\title{
Direct Drive Valve Model for Use as an Acoustic Source in a Network Model
}

\author{
Roel A. J. Müller and J. Hermann \\ IfTA Ingenieurbüro für Thermoakustik GmbH, 82194 Gröbenzell, Germany. \\ Wolfgang Polifke \\ Lehrstuhl für Thermodynamik, Technische Universität München, 85747 Garching, Germany.
}

Direct Drive Valves (DDVs) can be used as acoustic actuators in duct systems when requirements on mechanical or thermal robustness are high, e.g., for the active control of aerodynamic or combustion instabilities. This paper presents a model of a DDV that is used as an active element in an acoustic network model. In acoustic network modelling tools, acoustic sources are often implemented as simple velocity or mass flow boundary conditions. In practice, however, DDVs are not necessarily situated at the boundary of the system and the throughflow depends on the fluctuating pressure drop over the valve. This paper presents an acoustically compact model, based on mass conservation and a time-varying hydraulic resistance. The resistance depends on the fluctuating valve opening. The results are compared to the experiment in terms of acoustic wave transfer function.

\section{NOMENCLATURE}

$A$

$C_{d} \quad$ Discharge coefficient $[-]$

$D \quad$ Transmission coefficient of an acoustic delay $[-]$

$L \quad$ Length $[\mathrm{m}]$

$M \quad$ Mach number [-]

$Q \quad$ Gas flow rate in norm litre per minute [NLPM]

$R \quad$ Reflection coefficient [-]

$\mathcal{R}_{\text {sys }} \quad$ Combined reflection coefficient of an acoustic system $[-]$

Re Reynolds number [-]

$S \quad$ Source coefficient $[(\mathrm{m} / \mathrm{s}) / \%]$

$\mathcal{S}_{\text {sys }} \quad$ Combined source coefficient of an acoustic system $[(\mathrm{m} / \mathrm{s}) / \%]$

$T \quad$ Transmission coefficient $[-]$

$V \quad$ Volume $\left[\mathrm{m}^{3}\right]$

$c \quad$ Speed of sound $[\mathrm{m} / \mathrm{s}]$

$\tilde{f} \quad$ Downstream characteristic wave amplitude $[\mathrm{m} / \mathrm{s}]$

$\tilde{g} \quad$ Upstream characteristic wave amplitude $[\mathrm{m} / \mathrm{s}]$

$p \quad$ Pressure $[\mathrm{Pa}]$

$p_{0} \quad$ Total (stagnation) pressure $[\mathrm{Pa}]$

$r \quad$ Radius $[\mathrm{m}]$

$t \quad$ Time $[\mathrm{s}]$

$u \quad$ Velocity $[\mathrm{m} / \mathrm{s}]$

$x, y, z \quad$ Coordinates $[\mathrm{m}]$

$x_{\mathrm{sp}} \quad$ Valve (spool) opening [\%]

$\zeta \quad$ Hydraulic loss coefficient $[-]$

$\rho \quad$ Density $\left[\mathrm{kg} / \mathrm{m}^{3}\right]$

$\overline{0} \quad$ Time-averaged quantity

$\circ^{\prime} \quad$ Perturbation on time-averaged quantity

o $\quad$ Rate of change / flow $[0 / \mathrm{s}]$

\section{INTRODUCTION}

DDVs are generally used as hydraulic (oil flow) actuators. For this application, they need to be stiff and precise, but their range of operation is restricted to low frequencies. Recently, they have been used as acoustic (gas flow) actuators, such as for the active suppression of combustion instabilities or dynamic compressor stall. ${ }^{1-3}$

Combustion instabilities arise when there is a positive feedback between a fluctuating heat release and the combustor acoustics. These instabilities can quickly grow to great amplitudes, and in worst case, lead to severe hardware damage. Dampers, such as Helmholtz resonators, are often used to damp combustion instabilities, but for low frequencies (e.g. $<500 \mathrm{~Hz}$ ) the size of these dampers can get impractically large.

Active control by modulation of the fuel flow, is a commercial solution for these low-frequent instabilities. ${ }^{2}$ Compared to passive measures, active control on combustion and flow dynamics has the advantage of being more flexible. Different frequencies can be damped without hardware modification, and several modes of instability can be dealt with simultaneously. For a thorough overview on active and passive control on combustion instabilities, the reader is referred to $\mathrm{Zinn}^{4}$ and Culic. ${ }^{5}$

In these applications, valves, such as DDVs, have the advantage over loudspeakers that valves are very robust to thermal and mechanical influences. The volume flow modulated by a loudspeaker is limited by its geometry, while the modulated volume for a valve is limited by the throughflow. For the applications mentioned above, this is usually to the advantage of the valve.

The implementation of control systems as described before is not trivial; better tools are needed to predict the control authority a priori. ${ }^{6}$ Network models are a popular tool to analyse 1-D acoustics and could be a valuable aid for setting up new systems. However, until now, there has been no description of a valve being used for this type of model.

No truly acoustical description of how such a valve would need to be modelled was found in the literature. Annaswamy and Ghoniem, ${ }^{7}$ for instance, simply stated that the through- 
flow is assumed to be proportional to the valve opening, where the constant of proportionality depends, amongst others, on the (average) pressure drop over the valve. In reality, the throughflow depends on fluctuation of the pressure drop too. Wang et al. ${ }^{8}$ includes a description of the dynamic behaviour of the electro-mechanical subsystem of the valve, but does not discuss the resulting throughflow.

Reflection, transmission, and generation of acoustic waves by a constriction at high Mach numbers has been analyzed in the context of rocket exhaust nozzles. Contrary to the current work, it is usually assumed that the geometry of rocket nozzles is smooth and well known, and of course constant in time. Tsien, ${ }^{9}$ for instance, discussed a nozzle where the velocity $u$ is a linear function of position $x$. Candel, ${ }^{10}$ Marble and Candel, ${ }^{11}$ and Bohn ${ }^{12}$ generally assume smooth, conveniently defined geometries, where the only losses are caused by shocks. For valves on the other hand, losses are dominated by flow separation at edges. Mani, ${ }^{13}$ amongst others discussed, upstream reflection of free jets, but assumed that there was more knowledge of the flow field than is practically available for the flow through a commercial valve.

A model of a DDV is formulated and implemented in a linear network model of acoustic wave propagation and scattering. The DDV is described in Section 2, and the model will be formulated in Section 3. This model is implemented as an active element in taX, which is an acoustic network model, developed at the Technische Universitt Mnchen and implemented in MATLAB. ${ }^{14}$ The results are compared to experimental data in Section 4.

\section{DESCRIPTION OF THE PHYSICAL DDV}

The valve used in this research is DDV model D633E7320 by MOOG Inc. Valve models of this type are usually used for hydraulic applications. The manufacturer mentions metal forming and presses, automotive testing, and the timber industry. ${ }^{15}$ The control electronics of the valve under investigation were modified for use in active instability control of combustion instabilities, thus increasing its frequency range up to around $500 \mathrm{~Hz}$.

\subsection{Fluid-Mechanical Description}

Figure 1 gives a first impression of the outer appearance. A vessel (on top, largely outside of the picture) is connected to the inlet of the valve and functions as a decoupler, to acoustically decouple the valve from the upstream duct system. It implements, to a good approximation, an acoustically open boundary condition on the upstream side of the valve.

The valve itself, which is a cylinder type valve, is shown below the vessel. Only the bushing can be seen as a dark block on the photo, which is flanked by the linear motor on the left and the housing for electronics on the right.

The close-up below shows the principle gas flow and the spool in opened position. Moving the spool to the left would close the valve. The gas leaves the valve upward and then out through the exit duct, which points away from the observer.

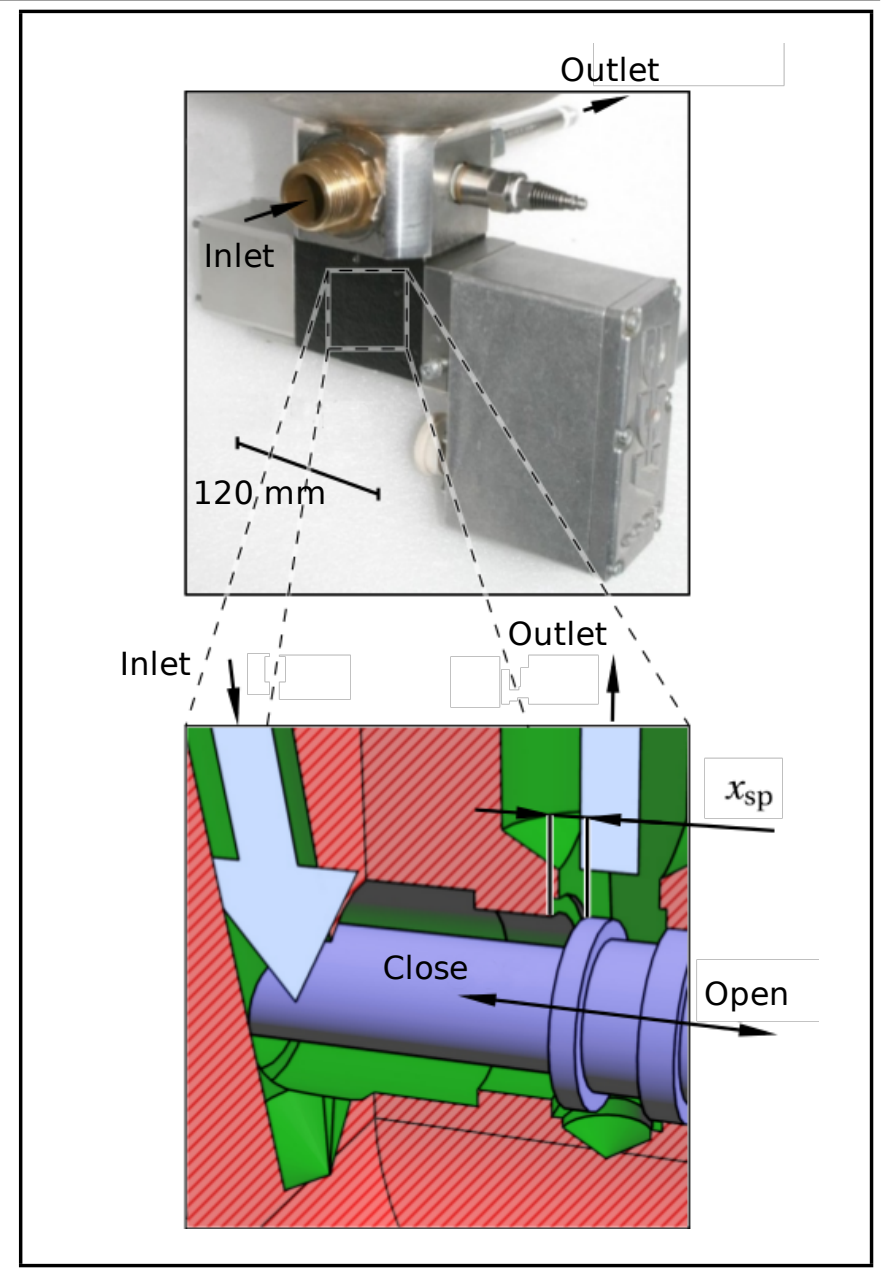

Figure 1. Overview of MOOG valve D633E7320, with a decoupler on top The close-up below shows the principle gas flow and the spool (blue) in opened position inside the bushing (green). Moving to the left, the spool would close the valve.

\subsection{Electro-Mechanical Description}

The spool position follows the input voltage as a second order dynamic system. Figure 2 gives a graphical impression. Unfortunately, there are a few non-linearities involved, which make it impractical to model the electro-mechanical system of the valve, as was done by Wang et al. ${ }^{8}$ in the linear network model taX. Most notably, hysteresis due to Coulomb friction causes significant, non-linear deviation from the ideal behaviour. The movement of the spool was restricted by hard ends (see Figs. 2 and 3). These introduce a clipping of the spool position for higher amplitudes. Since the spool position $x_{\mathrm{sp}}$ was monitored by a linear variable differential transformer (LVDT), a simpler model can be formulated using $x_{\mathrm{sp}}$ as input. Therefore, without loss of usability, the model will be restricted to the part indicated in the lower part of Fig. 2, i.e. concerning fluid mechanics only.

In typical industrial usage as an actuator controlling combustion instability of dynamic compressor stall, the mean spool position is half open $\left(x_{\mathrm{sp}}=50 \%\right)$. The spool oscillates harmonically around this position. The average flow is usually set by another valve in a slow feedback loop. This way, the DDV can remain half-open while the throughflow is varied. Hermann and Orthmann ${ }^{2}$ used the valve to modulate the fuel supply of the pilot flame of an industrial combustor. This con- 


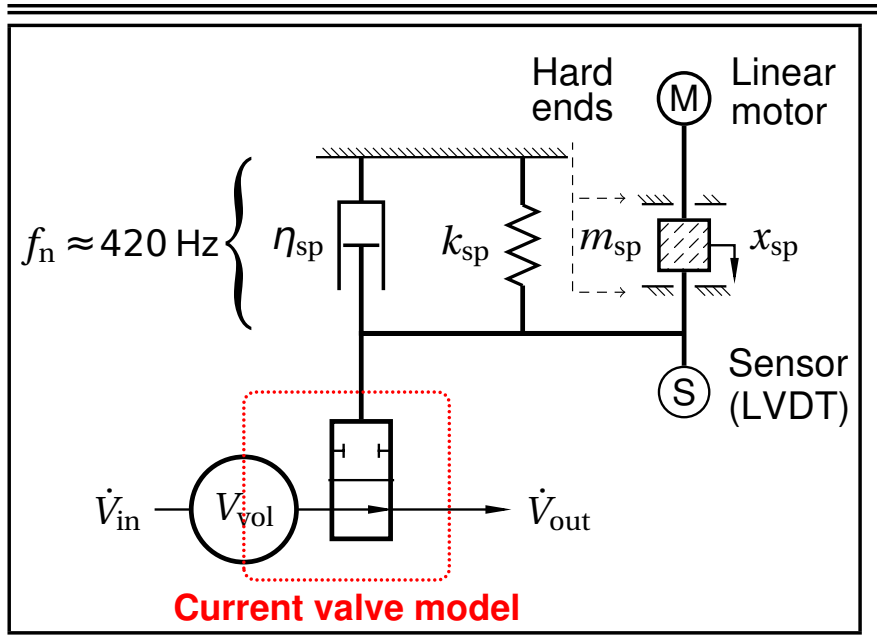

Figure 2. The dynamic system of the valve includes the most significant nonlinearities: friction (hysteresis) and hard ends limiting the movement of the spool.

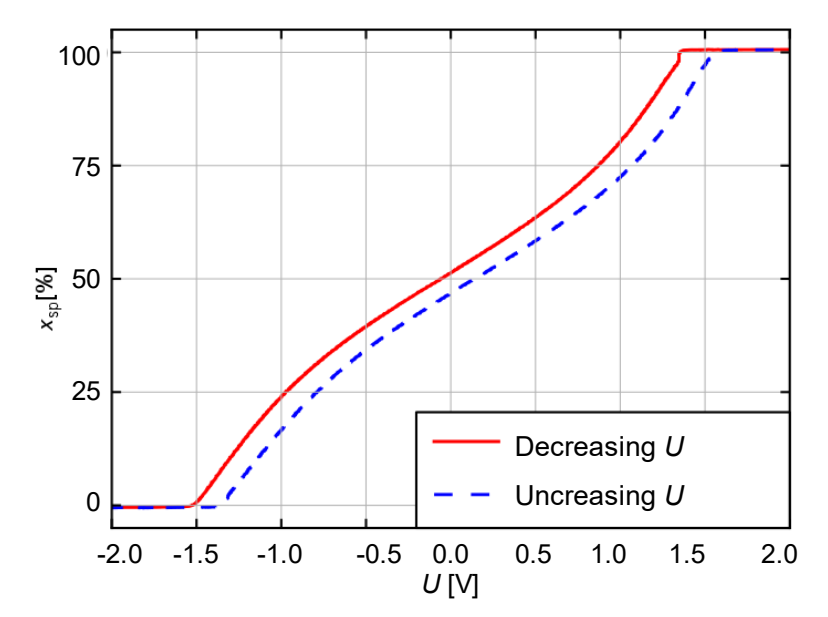

Figure 3. Hysteresis of (slowly varying) spool position as a function of input voltage. $x_{\mathrm{sp}}=0 \%$ and $x_{\mathrm{sp}}=100 \%$ are determined by the hard ends. At the $x_{\mathrm{sp}}=0 \%$ there is still some flow possible through the valve (see Section 3.2.1).

stituted only a relatively small fraction of the total amount of fuel burnt in the gas turbine. The amplitude of the actuation of the valve dependes on the instability at hand.

\section{LINEAR, COMPACT, QUASI STEADY, AND 1-D MODEL}

In the duct systems where DDVs are typically applied, acoustical effects can be considered quasi 1-D. Since the crossover frequency of the DDV lies around $500 \mathrm{~Hz}$, its application and the scope of the model considered here are limited to frequencies below this value. The wave lengths associated with these frequencies are of the order of (deci)metres, while the dimensions of the valve are limited to millimetres or centimetres. Therefore, the valve model will be formulated as acoustically compact.

The network modelling package taX deals with flow perturbation around a mean flow state. Quantities are written as a sum of a mean value and a perturbation. Mean values are indicated by an over-bar $(\bar{o})$, and perturbations by a prime $\left(o^{\prime}\right)$. The pressure becomes $p=\bar{p}+p^{\prime}$ and the velocity becomes $u=\bar{u}+u^{\prime}$, etc.

The frequency domain solver of taX uses models which are linear in perturbation. Therefore, the valve model as an element in taX must be linearised.

\subsection{The Mass Equation}

In the current valve, the volume of fluid inside the valve does not change under movement of the spool. Since the valve was acoustically compact as well, no mass was accumulated in the valve, and the mass flow on in- and outlet was the same $\left([\dot{m}]_{\text {out }}^{\text {in }}=[A \rho u]_{\text {out }}^{\text {in }}=0\right)$. Using the ideal gas law and Poisson's relation for calorically perfect gasses $\rho=\bar{\rho}+\rho^{\prime}=\bar{\rho}+p^{\prime} / c^{2}$, linearising and subtracting the mean equation gives: ${ }^{16}$

$$
\begin{aligned}
{\left[A\left(\bar{\rho} u^{\prime}+\bar{u} \rho^{\prime}\right)\right]_{\mathrm{in}}^{\mathrm{out}} } & =0+\mathcal{O}\left(\rho^{\prime} u^{\prime}\right) ; \\
{\left[A\left(\bar{\rho} u^{\prime}+\bar{u} \frac{p^{\prime}}{c^{2}}\right)\right]_{\mathrm{in}}^{\mathrm{out}} } & =0 ; \\
{\left[\bar{\rho} A\left(u^{\prime}+M \frac{p^{\prime}}{\bar{\rho} c}\right)\right]_{\mathrm{in}}^{\mathrm{out}} } & =0 .
\end{aligned}
$$

\subsection{The Momentum Equation}

The volume flow rate $\overline{\dot{V}}$ in a steady state was described by the discharge coefficient of the valve $\bar{C}_{d}$, where the outlet was taken as a reference point:

$$
\bar{u}_{\text {out }}=\frac{\overline{\dot{V}}_{\text {out }}}{A_{\text {out }}}=\bar{C}_{d} \sqrt{\frac{2}{\bar{\rho}_{\text {out }}}\left(\bar{p}_{0, \text { in }}-\bar{p}_{0, \text { out }}\right)} .
$$

\subsubsection{Measured steady discharge coefficient}

The pressure drop was measured* as a function of throughflow, for consecutive spool positions $x_{\mathrm{sp}}=10,20, \ldots, 100 \%$. The results, non-dimensionalised as the discharge coefficient $C_{d}$ and the Reynolds number $R e$, are plotted in Fig. 4. The discharge coefficient is weakly dependent on the flow rate. Under turbulent flow conditions, it is common for valves and orifices to show a power-law relation between flow rate and pressure drop. ${ }^{17,18}$ Therefore, it seems appropriate to express the variation of $C_{d}$ as a power function of Reynolds number $R e$.

According to the manufacturer's specifications, ${ }^{15}$ at a constant pressure drop, the steady state mass flow rate through the valve depends linearly on the spool position $x_{\mathrm{sp}}$. Constant pressure drops (where $R e$ is proportional to $C_{d}$ ) are found along lines through the origin of the axes, e.g., the diagonal dashed line in Fig. 4. If the curves $C_{d}(R e)$ for constant $x_{\mathrm{sp}}$ are individually curve-fitted by power-law functions, these cross the diagonal line at the points plotted in Fig. 5. This plot shows, that $C_{d}$ depends linearly on the spool position $x_{\mathrm{sp}}$, when the pressure drop is kept constant. Combining this linearity with the power-law behaviour gives the trend lines in Fig. 4.

\subsubsection{Unsteady momentum equation}

To use the valve model in an acoustic network model, two effects need to be considered. Firstly, the spool position should

${ }^{*}$ The loss in static pressure (not total pressure) was measured. The difference is insignificant for these speeds and pressure drops.

International Journal of Acoustics and Vibration, Vol. 21, No. 4, 2016 


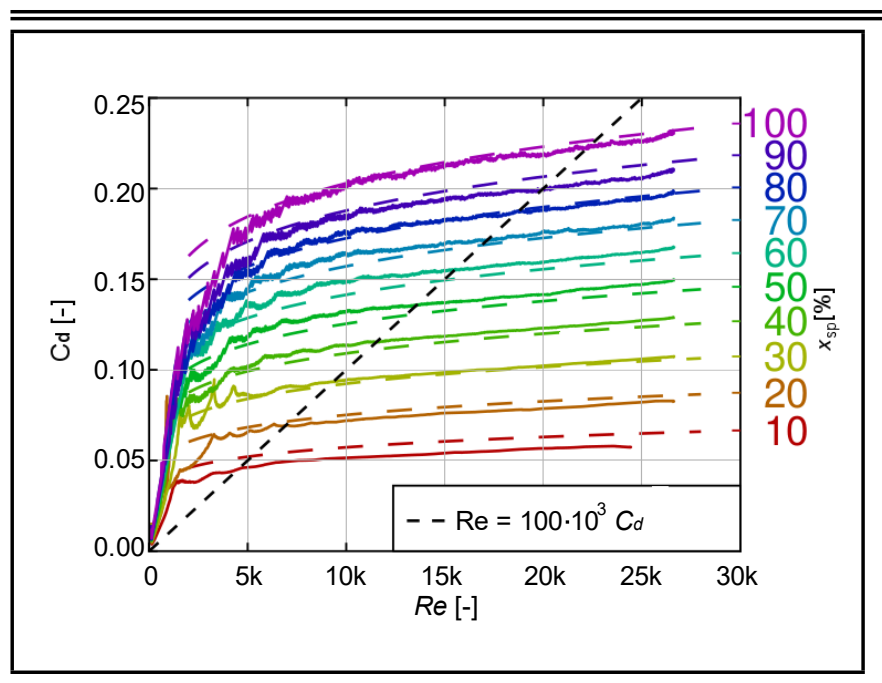

Figure 4. Trends of the discharge coefficient $C_{d}$ as a function of valve opening $x_{\mathrm{sp}}$ and throughflow, based on measurement data from. ${ }^{19}$ According to the specification by the manufacturer, at constant outlet pressure (the dashed diagonal line), $C_{d}$ should depend linearly on $x_{\mathrm{sp}}$.

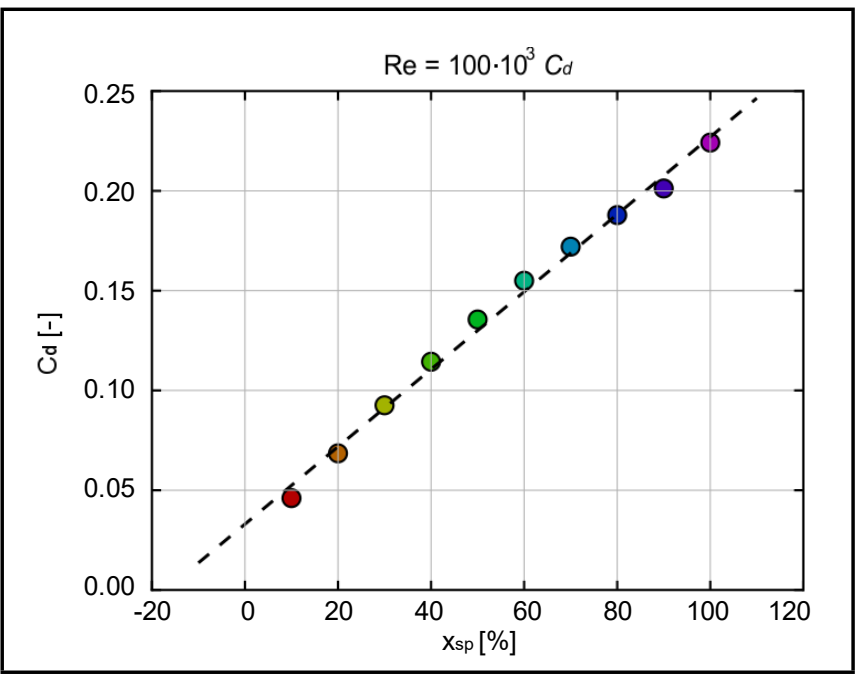

Figure 5. Trends of the discharge coefficient $C_{d}$ as function of the valve opening $x_{\mathrm{sp}}$ corresponding to the dashed diagonal line in Fig. 4. According to the manufacturer's specification, this should be a linear function. This is indeed the case, although there is an offset.

be allowed to oscillate around a mean value, so the valve can act as an acoustic source and when the valve is nearly closed, the flow accelerates through the contraction between the bushing and spool, which could be modelled as a lumped acoustical inertance after Hirschberg and Rienstra. ${ }^{20}$ Rewriting Eq. (2), with $\Delta p_{0}$ as a dependent variable and adding the inertia term leads to a formulation for the dynamic behaviour of the valve:

$$
\begin{aligned}
& \Delta p_{0}=p_{\text {in }}-p_{\text {out }}+\frac{1}{2} \rho_{\text {out }}\left(u_{\text {in }}^{2}-u_{\text {out }}^{2}\right)= \\
& \\
& C_{d}^{-2} \frac{\rho}{2} u^{2}+\rho L_{\kappa} \frac{\mathrm{d} u}{\mathrm{~d} t} ;
\end{aligned}
$$

where $C_{d}$ is now a quasi-steady function of time. Inertia is modelled by the term on the far right. The equivalent length $L_{\kappa}$ represents the length of a duct with the same amount of kinetic energy (for a certain throughflow) as the true geometry, but with constant cross section. Assuming homogeneous density throughout the constriction, $u(x)=\dot{m} /\left(\rho_{\text {ref }} A(x)\right) . L_{\kappa}$ can

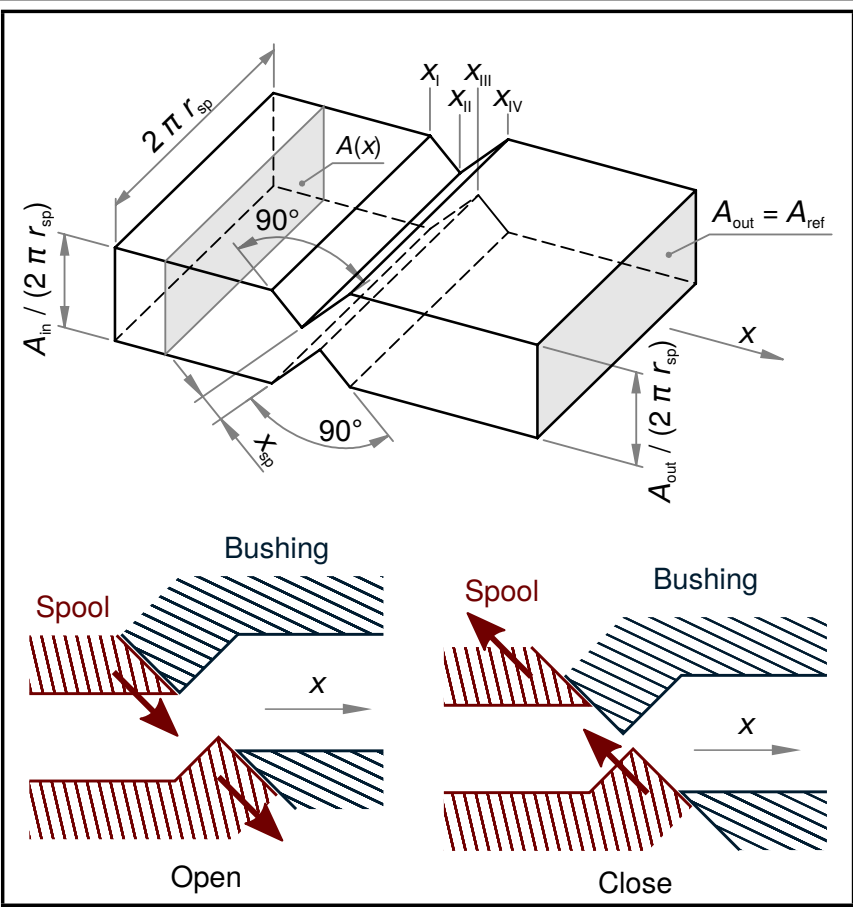

Figure 6. A simplified geometry was used for an approximation of the fluid at the constriction of the valve. The inlet and outlet ducts (subscript in and out) do not need to have an equal cross-sectional area.

now be expressed as:

$$
L_{\kappa}=\frac{\int \frac{1}{2} \rho_{\text {ref }} u(x)^{2} A(x) \mathrm{d} x}{\left[\frac{1}{2} \rho u^{2} A\right]_{\text {ref }}}=\int \frac{A_{\text {ref }}}{A(x)} \mathrm{d} x .
$$

Though the form of Eq. (4) is relatively simple, it requires an expression for the cross-sectional area as a function of streamwise coordinate $x$. Considering the geometry of the ducts in Fig. 1, this expression is not obvious. To find an approximation of $L_{\kappa}$, a simplified geometry was defined, as shown in Fig. 6.

This simplified geometry represents a "rolled out" version of the annular constriction between the spool and the surrounding bushing. It models the basic characteristics of the flow through the aperture of the valve; the flow converges at a $90^{\circ}$ angle while the ridges were offset by a distance of $x_{\mathrm{sp}}$. Also, opening and closing the valve did not change the volume of the fluid.

The contraction was modelled with zero volume, since the flow volume was modelled as part of the in-and outlet ducts. The additional inertia caused by the restriction, is therefore the inertia above the inertia represented by the in- and outlet ducts. Figure 7 expresses this concept graphically.

Closing the physical valve did not change the fluid volume. To keep the volume inside the simplified geometry constant as well, the contraction became longer when the valve was closed, and shorter when the valve was opened. This is shown in Fig. 8. The expression for the equivalent length $L_{\kappa}$, can be evaluated as:

$$
\begin{aligned}
& L_{\kappa}=L_{\kappa, \text { tot }}-L_{\kappa, \text { in }}-L_{\kappa, \text { out }}= \\
& A_{\text {ref }}\left(\int_{\mathrm{I}}^{\mathrm{IV}} \frac{1}{A(x)} \mathrm{d} x-\frac{x_{\mathrm{III}}-x_{\mathrm{I}}}{2 A_{\text {in }}}-\frac{x_{\mathrm{IV}}-x_{\mathrm{II}}}{2 A_{\text {out }}}\right)= \\
& \frac{A_{\text {ref }}}{4 \pi r_{\mathrm{sp}}} \ln \frac{A_{\text {in }} A_{\text {out }}}{A_{\text {min }}^{2}} .
\end{aligned}
$$




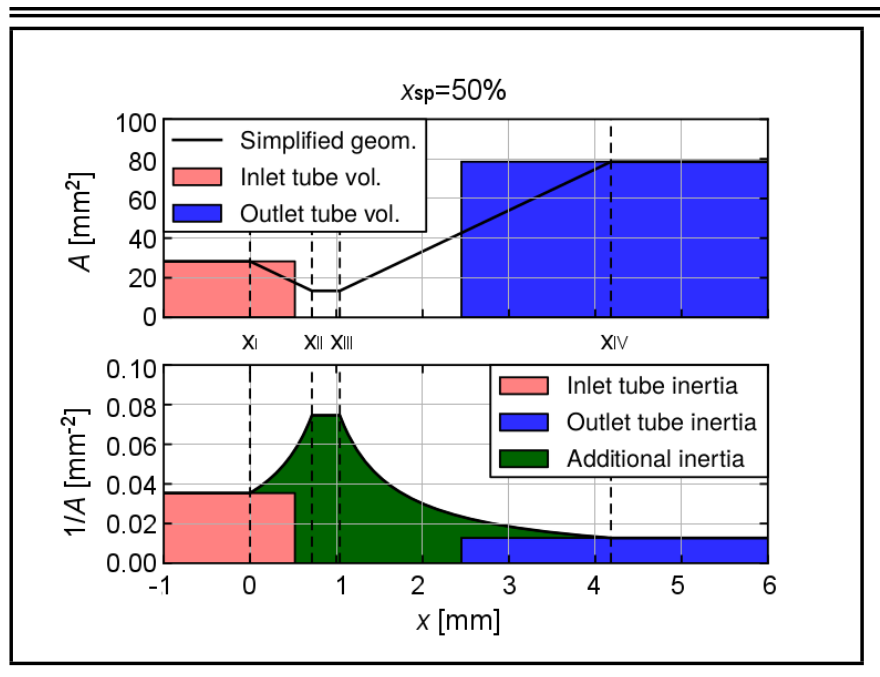

Figure 7. The volume of the fluid inside the in- and outlet duct of the valve was modelled by the elements representing those ducts. The additional inertia (compared to the undisturbed inertia of the inlet and outlet ducts) due to the constriction was added in terms of an equivalent length $L_{\kappa}$. The upper pane shows how the volume of the simplified valve geometry was redistributed amongst the inlet and outlet ducts. The lower pane shows the distribution of inertia of the flow.

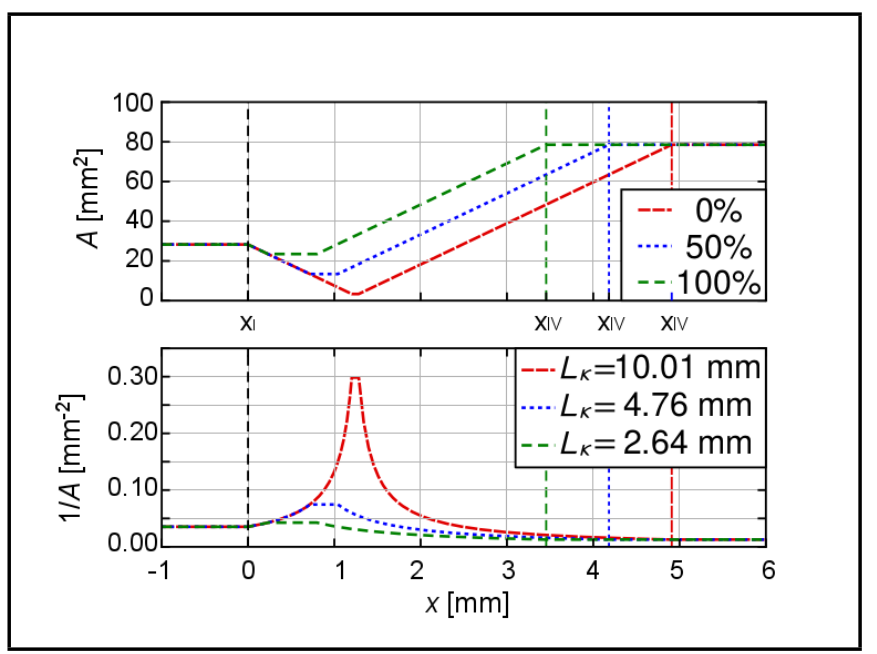

Figure 8. The influence of the degree of valve opening on $A(x)$ and $L_{\kappa}$.

In operation, the valve should not be fully closed, since then the flow could not be modulated any more. For the current DDV, $L_{\kappa}$ was estimated in the order of millimetres for all practical applications. In reality $L_{\kappa}$ would be somewhat larger due to the flow separation and jet formation, but still much smaller than the other relevant lengths in the system. Therefore, $L_{\kappa}$ can safely be neglected. For geometries where this inertia is relevant, it can be added as a separate element in the network model.

\subsubsection{Linearised fluctuating hydraulic resistance}

The formulations used in taX require components to be linearised and expressed in terms of the characteristic wave amplitudes $\tilde{f}=\frac{1}{2}\left(p^{\prime} /(\rho c)+u^{\prime}\right)$ and $\tilde{g}=\frac{1}{2}\left(p^{\prime} /(\rho c)-u^{\prime}\right)$. Firstly, the dynamic variables were expressed in the form of a steady mean value plus a fluctuation. This fluctuation was assumed to be small compared to the average, which meant that the terms of the order of fluctuations squared could be neglected.
By linearising $p=\bar{p}+p^{\prime}, \rho=\bar{\rho}+p^{\prime} / c^{2}, C_{d}=\bar{C}_{d}+C_{d}^{\prime}$, and $u=\bar{u}+u^{\prime}$, as well as leaving out the $L_{\kappa}$ term, Eq. (3) becomes:

$$
\begin{aligned}
& p_{\text {in }}^{\prime}+\bar{\rho}_{\text {in }} \bar{u}_{\text {in }} u_{\text {in }}^{\prime}= \\
& p_{\text {out }}^{\prime}-\bar{C}_{d}^{-2} \bar{\rho}_{\text {out }} \bar{u}_{\text {out }}^{2} \frac{C_{d}^{\prime}}{\bar{C}_{d}} \\
& +\left(1+\bar{C}_{d}^{-2}\right) \bar{\rho}_{\text {out }} \bar{u}_{\text {out }} u_{\text {out }}^{\prime}+\mathcal{O}\left(M^{2}, \text { pertubations }^{2}\right) .
\end{aligned}
$$

The steady solution can be subtracted from Eq. (6). Dividing the reminder by $\bar{\rho}_{\text {out }} c_{\text {out }}$, and substituting $\bar{C}_{d}{ }^{-2}$ by $\zeta$ and $\bar{u}$ byc $M$ gives:

$$
\begin{aligned}
& \begin{array}{l}
\underbrace{\frac{p_{\text {out }}^{\prime}}{\bar{\rho}_{\text {out }} c_{\text {out }}}}_{\text {pressure difference }}-\frac{p_{\text {in }}^{\prime}}{\bar{\rho}_{\text {out }} c_{\text {out }}} \\
+\underbrace{(1+\zeta) M_{\text {out }} u_{\text {out }}^{\prime}-\frac{\bar{\rho}_{\text {in }} c_{\text {in }}}{\bar{\rho}_{\text {out }} c_{\text {out }}} M_{\text {in }} u_{\text {in }}^{\prime}}_{\text {steady resistance, acceleration }}
\end{array} \\
& \underbrace{\zeta M_{\text {out }}^{2} c_{\text {out }} \frac{C_{d}^{\prime}}{\bar{C}_{d}}}_{\text {source term }} .
\end{aligned}
$$

The momentum and mass equations are rewritten as a matrix equation in terms of the characteristic wave amplitudes. The resulting matrix can then be used to characterise the valve element in the network model taX.

\section{COMPARING THE MODEL TO EXPERIMENT}

\subsection{Experimental Set-Up and System Model}

The acoustical behaviour of the valve was characterised by six transfer functions relating two outputs: upstream and downstream outgoing waves, to three inputs: upstream and downstream incoming waves, and actuation of the valve (see the block labelled "Valve element" in Fig. 9). Ideally, the model formulated before would be validated against an independent measurement of all these transfer functions. Unfortunately, this is not possible with the current valve, since there is no sensor access to the duct connecting the decoupler volume to the inlet of the valve, and this duct is impractically short to accommodate such access. The best that can be done is a determination of the reflection- and source coefficients of the system consisting of the valve combined with the inlet duct and decoupler volume. Figure 9 shows an overview of this system. The inlet duct was characterised by two transmission coefficients, $D_{\tilde{f}}$ and $D_{\tilde{g}}$, which were assumed to be pure delays. The reflection coefficient of the volume, $R_{V}$, was assumed to be $(1-M) /(1+M)$, based on acoustical energy conservation. The total reflection and source coefficients of this acoustical subsystem will be referred to as $\mathcal{R}_{\text {sys }}$ and $\mathcal{S}_{\text {sys }}$, respectively.

The model coefficients $T_{\tilde{f}}, T_{\tilde{g}}, R_{\text {in }}, R_{\text {out }}, S_{\text {in }}$, and $S_{\text {out }}$ were derived from the expressions derived from Eqs.(1) and (7). These coefficients were evaluated numerically, since the algebraic expressions would be unwieldily long. The full system 


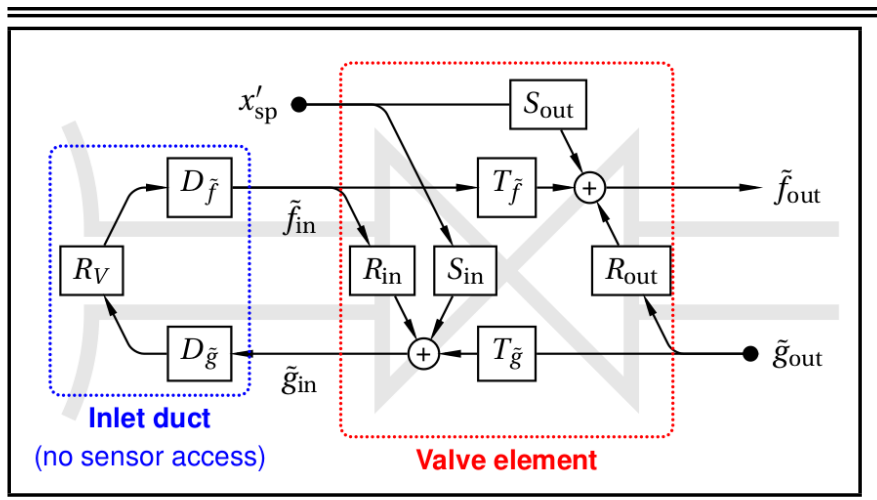

Figure 9. Overview of the acoustic transfer functions and the three measurable signals $\left(x_{\mathrm{sp}}^{\prime}, \tilde{f}_{\text {out }}\right.$ and $\left.\tilde{g}_{\text {out }}\right)$.

shown in Fig. 9 was described by the following matrix equation:

$$
\left[\begin{array}{ccccc}
\cdot & \left(D_{\tilde{g}} R_{V} D_{\tilde{f}}\right) & \cdot & \cdot & \cdot \\
R_{\text {in }} & \cdot & \cdot & T_{\tilde{g}} & S_{\text {in }} \\
T_{\tilde{f}} & \cdot & \cdot & R_{\text {out }} & S_{\text {out }}
\end{array}\right]\left[\begin{array}{c}
\tilde{f}_{\text {in }} \\
\tilde{g}_{\text {in }} \\
\tilde{f}_{\text {out }} \\
\tilde{g}_{\text {out }} \\
x_{\text {sp }}^{\prime}
\end{array}\right]=\left[\begin{array}{c}
\tilde{f}_{\text {in }} \\
\tilde{g}_{\text {in }} \\
\tilde{f}_{\text {out }}
\end{array}\right]
$$

where $\tilde{f}_{\text {out }}$ can be expressed as a function of $\tilde{g}_{\text {out }}$ and $x_{\mathrm{sp}}^{\prime}$ :

$$
\begin{aligned}
& \underbrace{\left(\frac{T_{\tilde{f}} D_{\tilde{g}} R_{V} D_{\tilde{f}} T_{\tilde{g}}}{1-R_{\text {in }} D_{\tilde{g}} R_{V} D_{\tilde{f}}}+R_{\text {out }}\right)}_{\mathcal{R}_{\text {sys }}} \tilde{g}_{\text {out }} \\
& +\underbrace{\left(\frac{T_{\tilde{f}} D_{\tilde{g}} R_{V} D_{\tilde{f}} S_{\text {in }}}{1-R_{\text {in }} D_{\tilde{g}} R_{V} D_{\tilde{f}}}+S_{\text {out }}\right)}_{\mathcal{S}_{\text {sys }}} x_{\text {sp }}^{\prime}=\tilde{f}_{\text {out }} .
\end{aligned}
$$

\subsection{Measurement and Signal Analysis}

The characteristic wave amplitudes were determined by the well known two-microphone method. ${ }^{21}$ In order to separate the source term and the reflection coefficient, two independent acoustical sources were needed. ${ }^{22}$ For this measurement, an additional loudspeaker was used. Figure 10 shows the experimental setup.

Introducing the superscript (ls) to identify quantities correlated to loudspeaker excitation, and (vl) to indicate quantities correlated to valve excitation, the two-state ansatz was written as the following matrix equation:

$$
\left[\begin{array}{c}
\tilde{f}_{\text {out }}^{(\mathrm{vl})} \\
\tilde{f}_{\text {out }}^{(\mathrm{ls})}
\end{array}\right]=\left[\begin{array}{cccc}
\mathcal{R}_{\text {sys }} & \mathcal{S}_{\text {sys }} & \cdot & \cdot \\
\cdot & \cdot & \mathcal{R}_{\text {sys }} & \mathcal{S}_{\text {sys }}
\end{array}\right]\left[\begin{array}{c}
\tilde{g}_{\mathrm{out}}^{(\mathrm{vl})} \\
x_{\mathrm{sp}}^{(\mathrm{vl})} \\
\tilde{g}_{\mathrm{out}}^{(\mathrm{ls})} \\
x^{\prime(\mathrm{l})} \\
\mathrm{sp}
\end{array}\right]
$$

The valve opening $x_{\mathrm{sp}}$ is constant when the loudspeaker is used for actuation, so $x_{\mathrm{sp}}^{\prime(\mathrm{ls})}=0$. Solving Eq. (10) gives the expres-

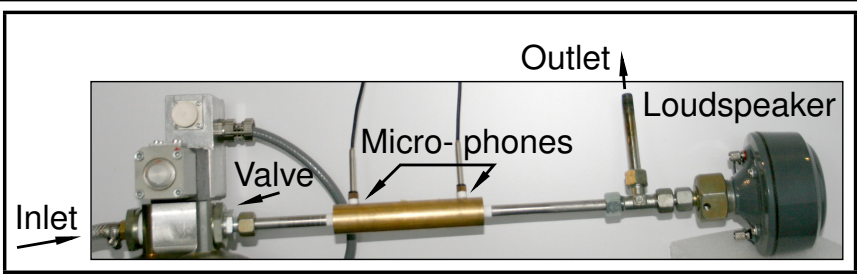

Figure 10. The experimental setup for the measurement of the reflection coefficient of $\mathcal{R}_{\text {sys }}$ and the source strength $\mathcal{S}_{\text {sys }}$ of the valve.

sions for $\mathcal{R}_{\text {sys }}$ and $\mathcal{S}_{\text {sys }}$ (similar to $\S 2.2$ in Bothien et al. ${ }^{23}$ ):

$$
\begin{aligned}
\mathcal{R}_{\mathrm{sys}} & =\frac{\tilde{f}_{\text {out }}^{(\mathrm{ls})}}{\tilde{g}_{\mathrm{out}}^{(\mathrm{ls})}} \\
\mathcal{S}_{\mathrm{sys}} & =\frac{\tilde{f}_{\mathrm{out}}^{(\mathrm{vl})}-\tilde{g}_{\mathrm{out}}^{(\mathrm{vl})} \mathcal{R}_{\mathrm{sys}}}{x_{\mathrm{sp}}^{\prime}} .
\end{aligned}
$$

\subsection{Cases}

Measurements were done with varying air mass flows, as well as with different values of the valve opening, which are listed in Table 1. For all cases, the transfer function relating the pressure fluctuation to the valve opening variation was determined in Pascal per percent opening fluctuation $(\mathrm{Pa} / \%)$. Subsequently, the two-microphone method ${ }^{21}$ was used to find the transfer function relating the characteristic wave amplitudes to the valve opening variation, from which the system coefficients were determined, as discussed before.

\subsection{Low-Frequency Limit}

In the quasi steady limit, and with $L_{\kappa}$ much smaller than relevant geometric lengths, Eqs. (1) and (3) are frequencyindependent. Since these equations don't contain any complex numbers, the acoustical coefficients describing the valve are real-valued functions of the steady conditions. Figure 11 shows how these quantities vary as a function of valve throughflow when the valve is opened to $50 \%$ on average. Figure 12 shows the variation as a function of valve opening, at 40 SLPM throughflow. The predicted low frequency limits of $\mathcal{R}_{\text {sys }}$ and $\mathcal{S}_{\text {sys }}$ (the quantities which can be measured experimentally for not too low frequencies) are given for comparison.

The influence of an increase in throughflow is similar to that of a decrease in opening. In both cases, the flow velocity through the constriction thus increases, increasing the acoustical stiffness of the valve.

When $A_{\text {in }}=A_{\text {out }}$ (not shown) all of the quantities vary between 0 and 1, except for $S_{\text {in }}$, which is negative. As far as the passive coefficients are concerned, this intuitively corresponds between the valve acting like a zero-length tube, a complete acoustical blockage, or something in between. The absolute values of the source coefficients accidentally were between 0 and 1 due to the choice of units. The source term's absolute value on the outlet side was slightly larger.

Currently, $r_{\text {in }}=3 \mathrm{~mm}$ and $r_{\text {out }}=5 \mathrm{~mm}$, so $A_{\text {in }}<A_{\text {out }}$. In this case, when the valve opening is large, or when there is little throughflow, the behaviour approaches that of an area discontinuity. For example $T_{\tilde{g}}$ can exceed unity and approache 2 as $A_{\text {in }} / A_{\text {out }}$ goes to zero $[24, \S 5.3] . R_{\text {in }}$ can be less than 


\begin{tabular}{|c|c|c|c|c|c|c|c|}
\hline \multirow[b]{2}{*}{ Case } & \multicolumn{2}{|c|}{ Flow rate } & \multirow[t]{2}{*}{ Opening } & \multirow[t]{2}{*}{$\bar{C}_{d}$} & \multirow{2}{*}{$\begin{array}{c}\Delta p \\
(\mathrm{~Pa})\end{array}$} & \multirow[t]{2}{*}{$M_{\text {out }}$} & \multirow[t]{2}{*}{$\operatorname{Re}$} \\
\hline & SLPM & $\mathrm{g} / \mathrm{s}$ & & & & & \\
\hline Q020-X050 & 20 & 0.33 & $50 \%$ & 0.106 & 1091 & 0.0132 & 3236 \\
\hline Q040-X025 & \multirow{3}{*}{40} & \multirow{3}{*}{0.67} & $25 \%$ & 0.077 & 8207 & \multirow{3}{*}{0.0263} & \multirow{3}{*}{6471} \\
\hline Q040-X050 & & & $50 \%$ & 0.117 & 3609 & & \\
\hline Q040-X075 & & & $75 \%$ & 0.154 & 2073 & & \\
\hline Q080-X050 & 80 & 1.33 & $50 \%$ & 0.128 & 11940 & 0.0526 & 12943 \\
\hline Q160-X050 & 160 & 2.67 & $50 \%$ & 0.141 & 39500 & 0.1053 & 25885 \\
\hline
\end{tabular}

Table 1. The overview of the investigated operating conditions.
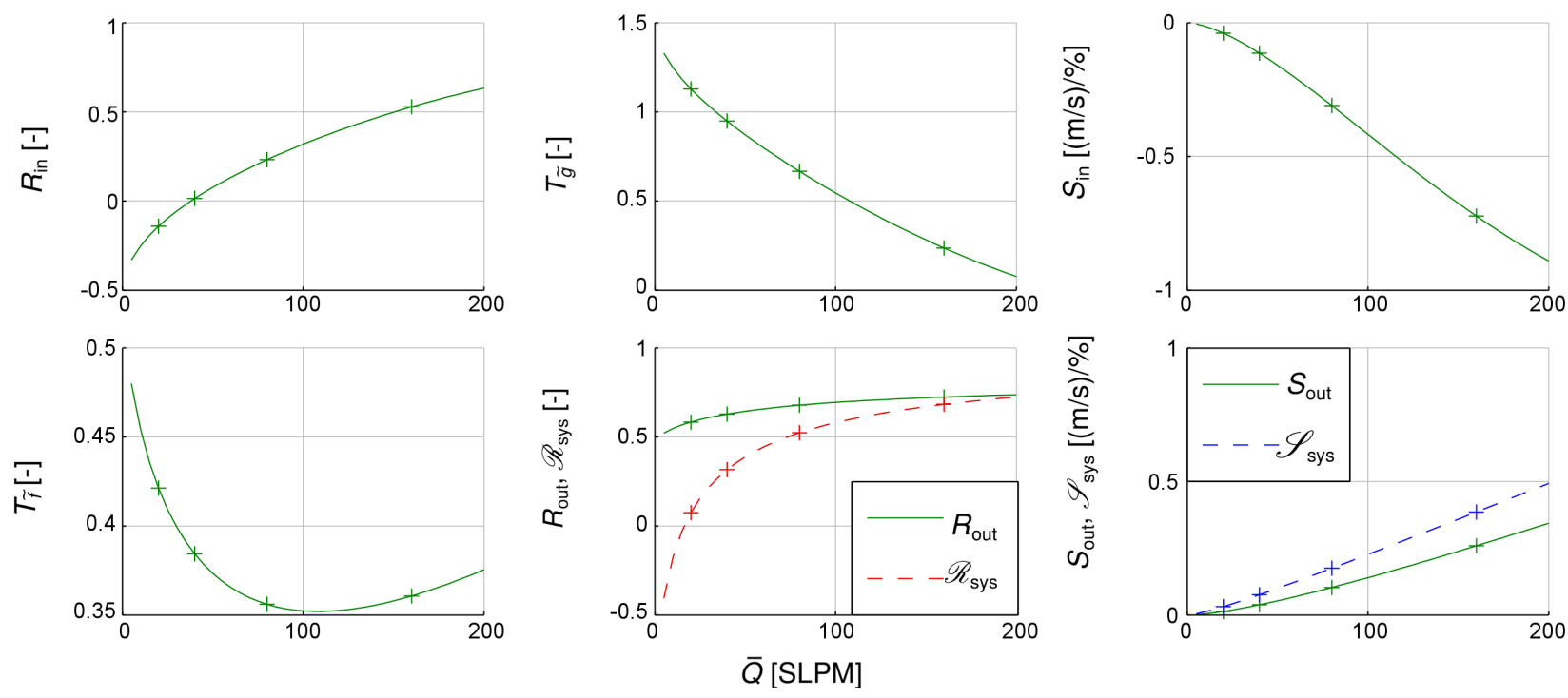

Figure 11. Modelled transfer functions, according to Eqs. (1) and (7), as functions of the throughflow $\bar{Q}$ in SLPM (standard litre per minute). Mean valve opening $\bar{x}_{\mathrm{vl}}$ was $50 \%$. The markers indicate operating points listed in Table 1.
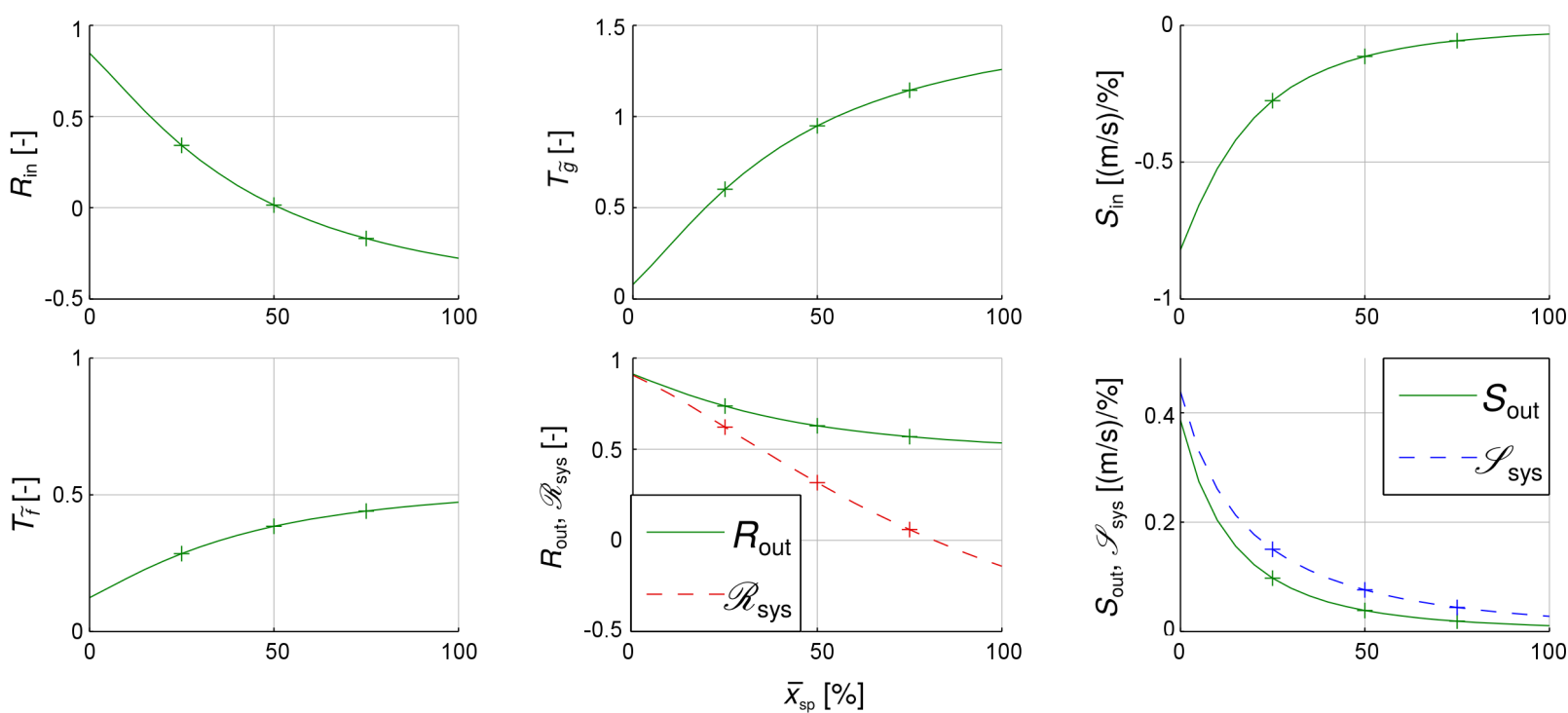

Figure 12. Modelled transfer functions, according to Eqs. (1) and (7), plotted against the mean valve opening $\bar{x}_{\text {sp }}$ in percentage. Throughflow $\bar{Q}$ was 40 SLPM. The markers indicate operating points listed in Table 1. 

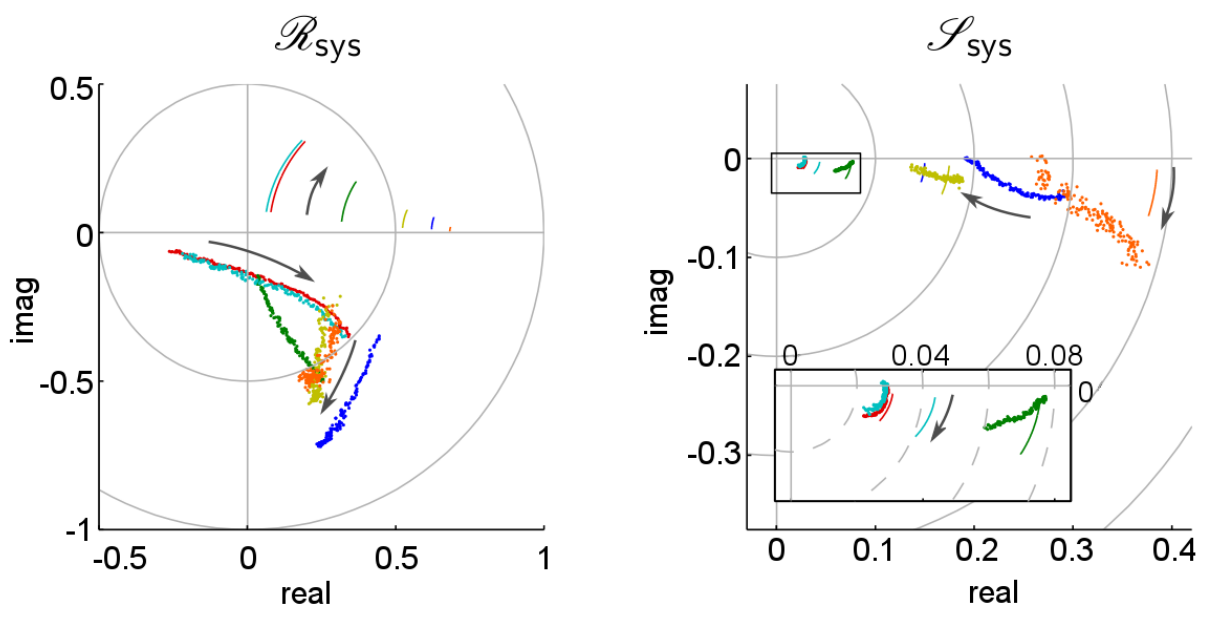

- Model

$\therefore$ Measurement

Q020-X050

- Q040-X025

- Q040-X050

- Q040-X075

Q080-X050

Q160-X050

Figure 13. Reflection (left/below) and source term (right/above) of the system plotted in the complex plane, for frequency in the range $f \in[100,500] \mathrm{Hz}$. The arrows indicate the direction of frequency dependence. $\mathcal{R}_{\text {sys }}$ is dimensionless and $\mathcal{S}_{\text {sys }}$ is measured in $(\mathrm{m} / \mathrm{s}) / \%$.

zero, when the combined impedance - of the valve plus the outlet duct - is less than the impedance of the inlet duct. The source term was greater on the side with the larger diameter.

\subsection{Frequency Dependence}

Figure 4.4 gives an overview of the measured reflection coefficient and source term compared to the prediction. The lower limit of the frequency range for this measurement was caused by the loudspeaker, which did not excite efficiently enough below $100 \mathrm{~Hz}$. The high frequency limit of the measurement was determined by the valve, which could only be operated up to around $500 \mathrm{~Hz}$.

The predicted reflection coefficient, as function of frequency, began at the positive real axis and curved up to the right. Intuitively, this could be understood as a direct reflection form of the valve, plus a delayed open end; i.e., the reflection of the decoupler. The measured reflection coefficient shows similar trends depending on the operating condition; the reflection increases as the valve is closed, or as throughflow is increased. The low-frequency limit, which can be evaluated better from the bode plots in Figs. 4.5 and 4.5, lie on the real axis. For the cases Q020-X050 and Q040-X075, the reflection coefficient at low frequencies was predicted to be positive, while the measurements showed a negative value. The phase was generally lower then predicted, and fell further as the frequency increased. The reason for this behaviour remains unknown.

The low-frequency limit of the source term is situated on the positive real axis; the flow increases when the valve is opened. Reflected waves from the decoupler have the same sign with a small delay and cause the curve to go down to the left as the frequency increased. The magnitude of the source term increases when the valve was closed, or when throughflow was increased. The predicted source term shows a better agreement with the experiment than the reflection coefficient. The main discrepancies were that the measured source terms showed a greater dependence on valve opening, and, in some cases, another behaviour for increasing frequency.

\section{IMPLEMENTATION IN A SIMPLIFIED FUEL SYSTEM}

\subsection{Set-up}

To demonstrate the usability of the valve model in an acoustic network model, the setup in Fig. 10 was modified. After the removal of the tee-piece and the loudspeaker, what remains can be thought of as a simplified fuel system. The representation of this system in the network modelling programme taX is shown in Fig. 16. An open inlet represents the decoupler. The valve element (labelled "DDV") was accompanied by short ducts representing the inlet and outlet inside the bushing and decoupler. Additional inertia at the valve outlet was set to zero, as argued in Section 3.2.2, and was included for illustrative purposes. Ducts $\mathrm{A}, \mathrm{B}$, and $\mathrm{C}$ together form the simplified fuel supply system. The two microphones measured the pressure fluctuation caused by the valve as before.

\subsection{Results}

The predicted and measured transfer functions are shown in Figs. 17 and 18. Since the valve was predicted to behave similar to a closed end, and the exit was open, the resonances corresponded to the $1 / 4$ and $3 / 4$ wave modes of the duct. For Q020-X050 and Q040-X075, where the valve, contradictory to the model, behaved more like an open end, the measured peaks in the transfer function were much more damped.

In general, the model captures the order of magnitude of the sound generated by the valve well. It should be noted that the valve can hardly be operated above $500 \mathrm{~Hz}$, so the deviation form prediction at higher frequencies was not dramatic for application.

\section{COMPARISON TO THE LOUDSPEAKER}

As argued in Section 4.1, not all coefficients describing the valve model could be measured independently. Nonetheless, the current data was sufficient enough to make a comparison between a valve and a loudspeaker as acoustic actuators. 


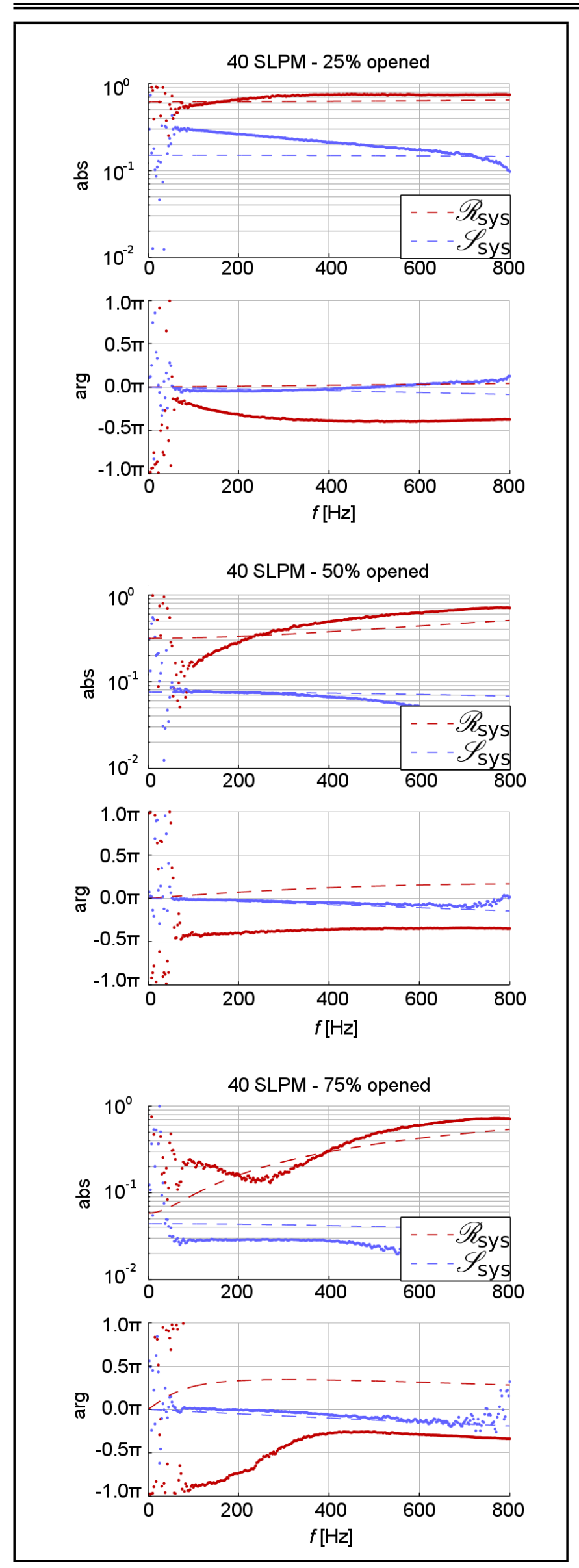

Figure 14. The model prediction of the valve's transfer function (dashed), compared to measurement (dots) for various values of average valve opening. $\mathcal{S}_{\text {sys }}$ is in $(\mathrm{m} / \mathrm{s}) / \%$ and $\mathcal{R}_{\text {sys }}$ is dimensionless.
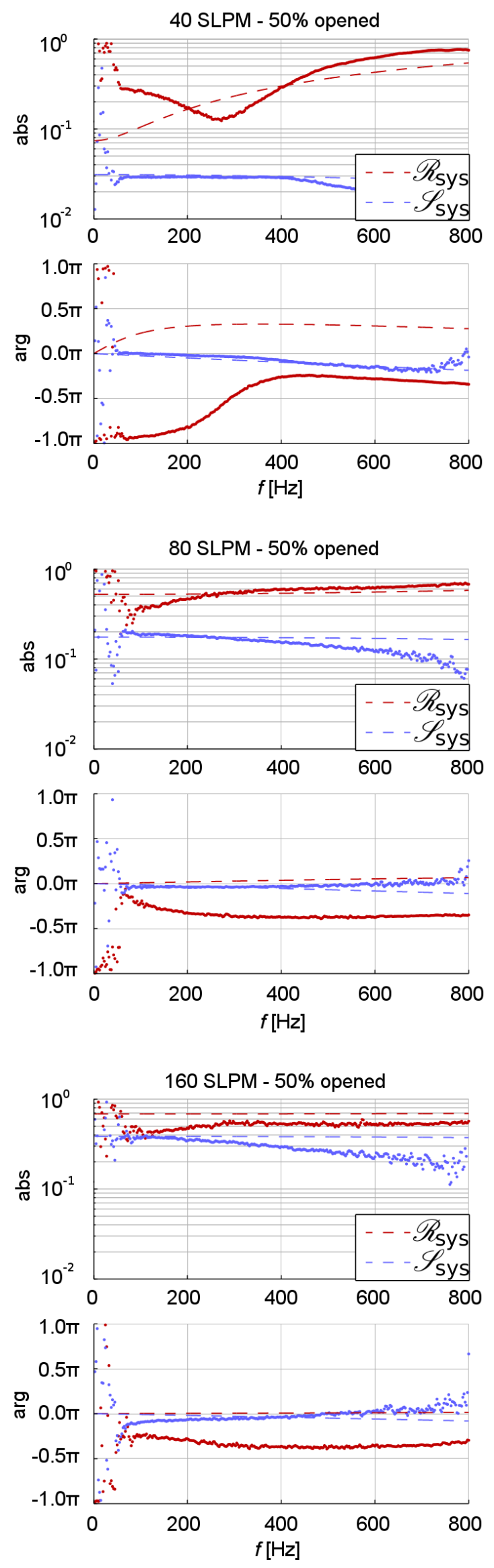

Figure 15. The model prediction of the valve's transfer function (dashed), compared to the measurement (dots) for various average mass flow rates in standard litre per minute (40 SLPM is shown in Fig. 4.5). $\mathcal{S}_{\text {sys }}$ is in $(\mathrm{m} / \mathrm{s}) / \%$ and $\mathcal{R}_{\text {sys }}$ is dimensionless. 


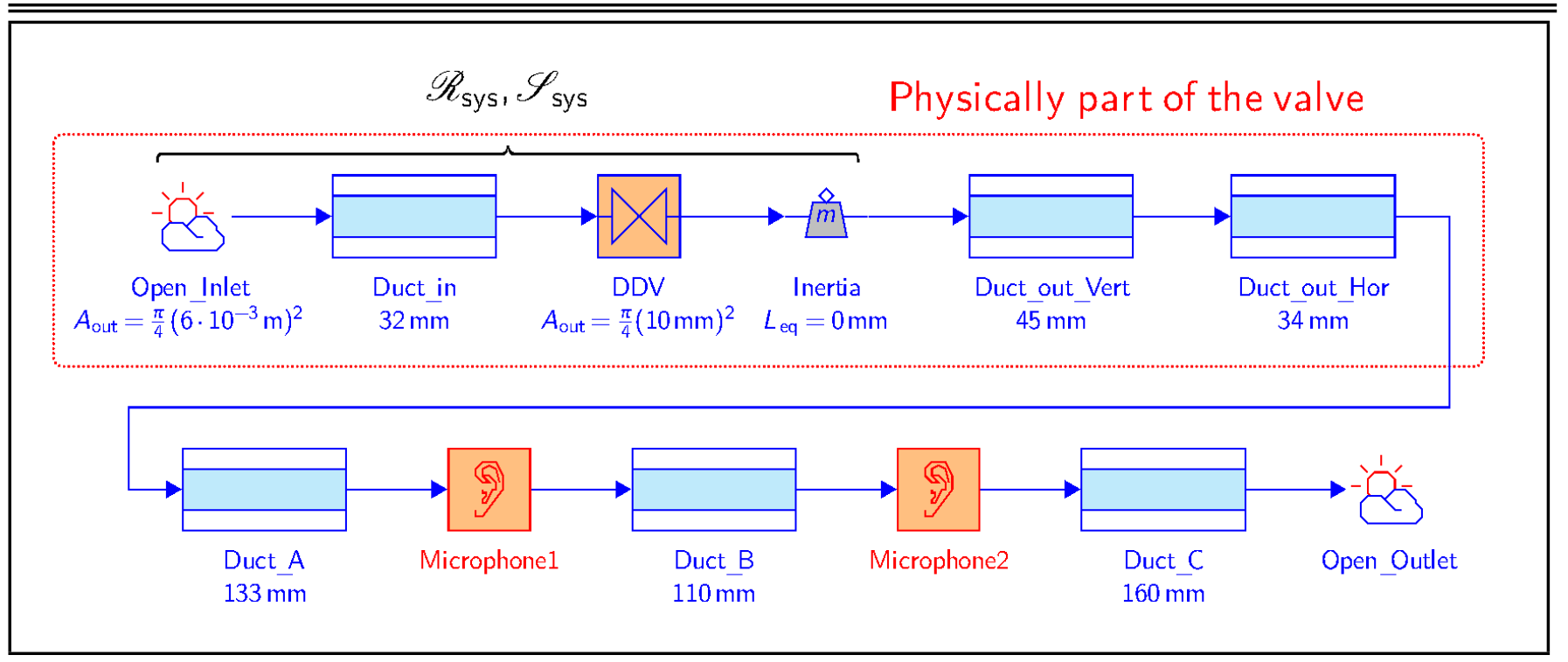

Figure 16. Representation of the simplified fuel system in taX: the elements in the upper half of the taX model together represent the valve and decoupler. A simple duct with two microphones in the lower half complete this simplified fuel supply system.

In acoustical network modelling, loudspeakers are often assumed to behave like fluctuating volume sources, since they are "much stiffer" than the medium involved and do not react to pressure fluctuations. ${ }^{7,14}$ Although more advanced models exist, $^{25-27}$ in the first approximation for many relevant situations, a loudspeaker mounted at the end of a duct could be assumed to behave like a hard end and a constant source term in a superposition.

The behaviour of the valve introduces much more damping. Figure 4.4 shows reflection coefficients that vary between unity and zero. The measured reflection coefficient of the valve with the decoupler was negative for some operating conditions.

While the influence of the flow conditions on a loudspeaker are usually neglected, the valve behaved differently depending on the conditions. Both reflection and sound creation increased with throughflow or reduction of the opening.

Finally, from a practical perspective, the volume modulated per cycle by a loudspeaker was limited by its geometry. For a valve, on the other, hand the modulated volume was limited by its throughflow. Since throughflow per cycle is greater for lower frequencies, a valve was a more effective actuator at lower frequencies.

When an upstream duct is modelled, another relevant difference between both actuators is that the loudspeaker behaves like a volume source, i.e., an acoustic monopole. The loudspeaker, on the other hand, modulates the pressure drop, and hence acts as an acoustic dipole.

\section{DISCUSSION AND CONCLUSIONS}

The model presented in this paper describes the basic characteristics of a DDV. Embedded in an acoustic network, it can give a good first prediction of the effectiveness of the implementation, i.e., the control authority of the valve.

There remains a significant discrepancy in the prediction of the phase of the reflection coefficient $\mathcal{R}_{\text {sys }}$, as shown in Figs. 4.4, 4.5 and 4.5. This discrepancy was at its largest for the low-frequency limit of the cases Q020-X050 and Q040-X075, where the absolute value of the reflection coefficient was relatively small. For other cases and frequencies, the phase difference was less, but often around $\pi / 2$.

Equation (9) gives $\mathcal{R}_{\text {sys }}$ as the sum of $R_{\text {out }}$ and another term, that involved the reflection at the decoupler, amongst others. Since both terms were nearly opposite in phase according to the model, small errors in either one of the terms, could lead to large phase errors in $\mathcal{R}_{\text {sys }}$. It cannot be determined from the current measurements which of the quantities in Eq. (9) caused the discrepancy because the valve assembly discussed in this paper did not allow for measurements of the acoustics upstream of the valve.

The assumption that the decoupler behaved as an open end did not cause the discrepancy. Network simulations with a Helmholtz resonator instead of an open ended did not yield a better agreement.

Furthermore, an acoustical influence of the corners and cross-sectional variation of the ducts inside the bushing of the valve was hard to predict and even harder to separate from other effects. For further improvement of the model, it would be advisable to test the model on a valve with a cleaner and more accessible duct geometry.

This discussion does not differentiate between $M$ and $R e$ effects, since only one fluid (air) was used. The model could be improved, by redoing the $\bar{C}_{d}$ measurements with another gas, which is especially true for applications in fuel gas supply systems.

The (linear) model assumes the modulation of the discharge coefficient $C_{d}^{\prime}$, which appears in the source term, to be small compared to the mean value $\bar{C}_{d}$. When $C_{d}^{\prime}$ is increased to improve control authority over hard-to-control combustion instabilities, this assumption, needed for the linearisation, no longer holds.

The current model can be used in the initial stage of implementing a system for rapid flow control, and as such, the model can help speeding up this phase of development. 


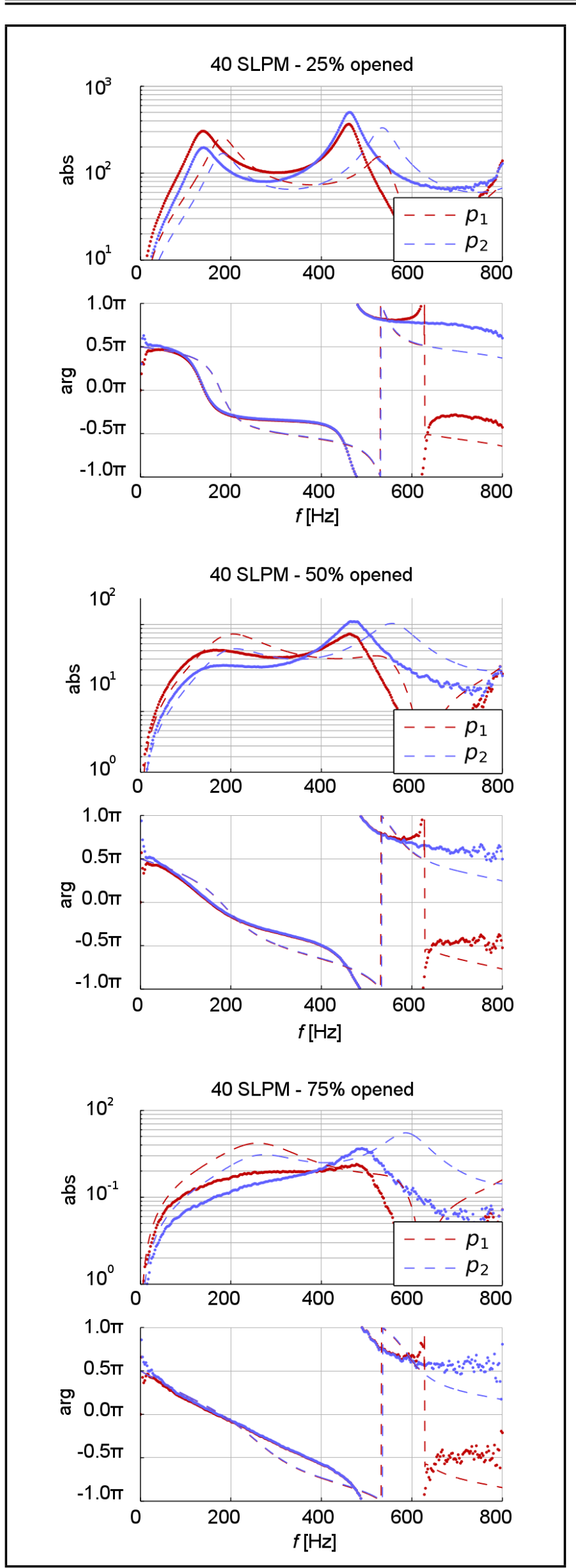

Figure 17. Model prediction of the valve's transfer function (dashed), compared to the measurement (dots) for the various values of the average valve opening.
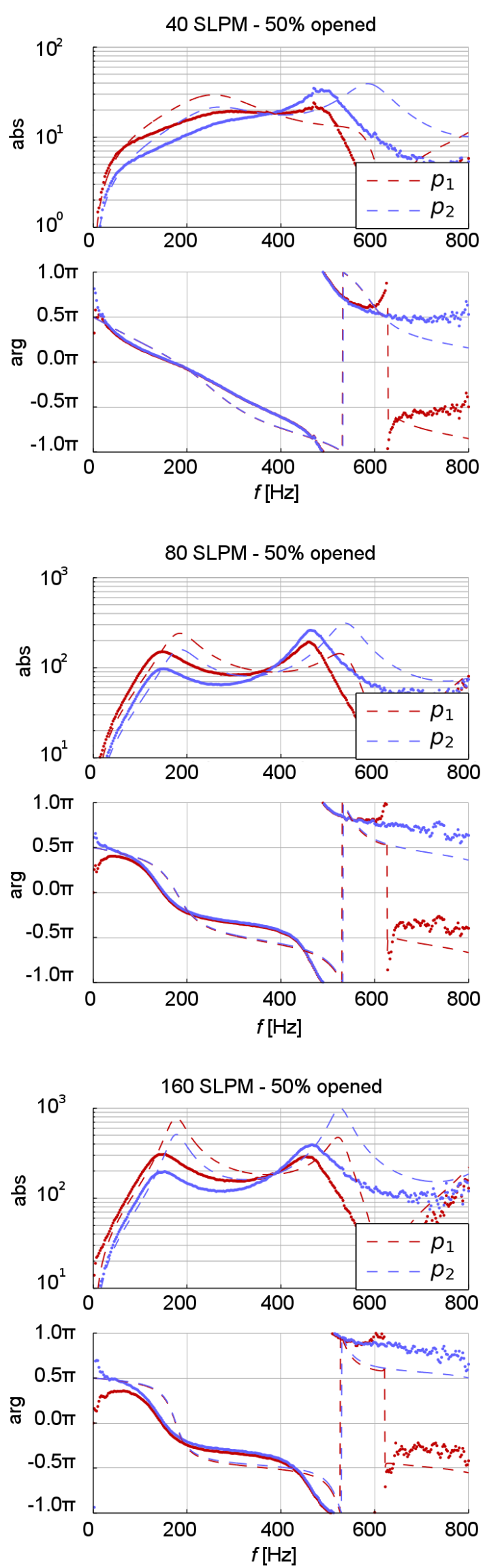

Figure 18. The model prediction of the valve's transfer function (dashed), compared to the measurement (dots) for the various average mass flow rates in standard litre per minute (40 SLPM is shown in Fig. 17). 


\section{ACKNOWLEDGEMENTS}

This work was done for the EU-funded Marie Curie research project LiMOUSINE (FP7-214905). The financial support from the European commission is gratefully acknowledged.

\section{REFERENCES}

1 Schadow, K. C. and Ken, H. Y. Active combustion control: Recent R\&D and outlook for implementation. Int'l Symp. Air Breathing Engines (ISABE), number ISABE2001-1018, (2001).

2 Hermann, J. and Orthmann, A. Combustion dynamics: Application of active instability control on heavy duty gas turbines. RTO / AVT Course "Active Control of Engine Dynamics”, (2001). published in RTO-EN-020.

3 Hiller, S. J., Hermann, J. and Widhopf-Fenk, R. High pressure compressor stabilization by controlled pulsed injection. ASME Turbo Expo 2012, GT2012-68460, Copenhagen, DK, (June 2012).

4 Zinn, B. T. Smart combustors - just around the corner. ASME Turbo Expo, GT2005-69138, (2005).

5 Culick, F. E. C. Unsteady motions in combustion chambers for propulsion systems. Technical Report RTO-AG-AVT039, RTO / NATO, (2006).

${ }^{6}$ Culick, F.E.C. and Palm, S. Active control of combustors after twenty years' efforts. Progr. in Propulsion Physics, 1 441-468, (2009). http://dx.doi.org/10.1051/eucass/200901441

7 Annaswamy, A. M. and Ghoniem, A. F. Active control of combustion instability: Theory and practice. Control Systems, IEEE, 22 (6), 37-54, (2002).

8 Wang, Y., Megli, T., Haghgooie, M., Peterson, K. S., and Stefanopoulou, A. G. Modeling and control of electromechanical valve actuator. Soc. Automotive Engineers, Inc., SAE 2002-01-1106, (2002).

9 Tsien, H.S. The transfer function of rocket nozzles. J. American Rocket Soc., 22 139-143, (1952).

10 Candel, S. M. Acoustic conservation principles and application to plane and modal propagation in nozzles and diffusers. J. Sound \& Vibration, 41(2), 207-232, (1975).

11 Marble, F. E. and Candel, S. M. Acoustic disturbance from gas non-uniformities convected through a nozzle. J. Sound \& Vibration, 55(2), 225-243, (1977).

12 Bohn, M.S. Response of a subsonic nozzle to acoustic and entropy disturbances. J. Sound \& Vibration, 52(2), 283-297, (1977). ISSN 0022-460X. http://dx.doi.org/10.1016/0022-460X(77)90647-2.

13 Mani, R. Low-frequency sound propagation in a quasi-onedimensional flow. J. Fluid Mech., 104(1):81-92, (1981). http://dx.doi.org/10.1017/S0022112081002826
14 Leandro, R., Huber, A., and Polifke, W.. taX manual. Technische Universität München, v0.9 edition, (October 2010).

15 MOOG Inc. Direct-operated servo valves for ana$\log$ signals D633 and D634 series. Website, April 2013. URL http:// www.moog.com/products/servovalvesservo-proportional-valves/ industrial/flow-control/analogwith-integrated-electronics/ direct-operated-servo-valvesfor-analog-signals-d633-d634-series.

16 Polifke, W. Combustion instabilities. Advances in acoustics and applications, Brussels, Belgium, (2004). VKI Lecture series.

17 Crane Co. Technical Report 410, Flow of fluids through valves, pipes, and fittings. Crane Co., Chicago, US-IL, (1972). 12th printing, 2-7.

18 Rhinehart, R. R., Gebreyohannes, S., Sridhar, U. M., Patrachari, A., and Rahaman, M. S. A power law approach to orifice flow rate calibration. ISA Trans., 50, 329-341, (2011).

19 Kaiser, T. Experimentelle Untersuchung von ServoLinearmotor-Ventilen der Firma Moog. Technical report, IfTA Ingenieurbüro für Thermoakustik GmbH, (2012).

20 Hirschberg, A. and Rienstra, S. W. Elements of aeroacoustics. Lecture Series 1994-04. Von Karman Institute for Fluid Dynamics, (1994).

21 Seybert, A.F. and Ross, D.F. Experimental determination of acoustic properties using a two-microphone randomexcitation technique. J. Acoustical Soc. America, 61(5), 1362-1977, (May 1977).

22 Munjal, M. L. and Doige,A. G. Theory of a two sourcelocation method for direct experimental evaluation of the four-pole parameters of an acoustic element. J. Sound \& Vibration, 141, 323-333, (1990).

23 Bothien, M. R., Moeck, J.P., and Paschereit, C. O. Active control of the acoustic boundary conditions of combustion test rigs. J. Sound \& Vibration, 318 678-701, (2008). http://dx.doi.org/10.1016/j.jsv.2008.04.046

24 Hirschberg, A. Introduction to aero-acoustics of internal flows. Revised version of chapter from the course Advances in Aeroacoustics (VKI, 12-16 March 2001), (2009).

25 Thiele, A. N.. Loudspeakers in vented boxes. J. Audio Eng. Soc., 19-20, 382-392, 471-483, (1971).

26 Small, R. H.. Direct-radiator loudspeaker system analysis. J. Audio Eng. Soc., 21(5), 363-372, (1973).

27 Marx, D., Mao, X. and Jaworski, A. J. Acoustic coupling between the loudspeaker and the resonator in a standing-wave thermoacoustic device. Applied Acoustics, 67(5), 402-419, (2006). http://dx.doi.org/10.1016/j.apacoust.2005.08.001 